\title{
Impact of pathological complete response following neoadjuvant chemoradiotherapy in esophageal cancer
}

\author{
Tamer Soror $^{1,2}$, Gerlinda Kho ${ }^{1}$, Kuai-Le Zhao $^{3}$, Mahmoud Ismail ${ }^{4}$, Harun Badakhshi ${ }^{1}$ \\ ${ }^{1}$ Department of Clinical Radiation Oncology, Ernst von Bergmann Medical Center, Academic Teaching Hospital of Humboldt University Berlin \\ (Charité), Berlin, Germany; ${ }^{2}$ Radiation Oncology Department, National Cancer Institute (NCI), Cairo University, Cairo, Egypt; ${ }^{3}$ Department of \\ Radiation Oncology, Fudan University Shanghai Cancer Center, Shanghai 200032, China; ${ }^{4}$ Department of Surgery, Competence Center of Thoracic \\ Surgery, Charité - Universitätsmedizin Berlin, Berlin, Germany \\ Contributions: (I) Conception and design: T Soror, H Badakhshi; (II) Administrative support: G Kho; (III) Provision of study materials or patients: H \\ Badakhshi; (IV) Collection and assembly of data: T Soror; (V) Data analysis and interpretation: T Soror; (VI) Manuscript writing: All authors; (VII) \\ Final approval of manuscript: All authors. \\ Correspondence to: Tamer Soror. Winfried Str. 2, 14169 Berlin, Germany. Email: Tamer.soror@nci.cu.edu.eg.
}

Background: Neoadjuvant radiochemotherapy (nRCT) followed by surgery has become the gold standard treatment in patients with locally advanced esophageal cancer. The pathological response is an important predictor in such patients. This work represents a single-center analysis investigating the impact of pathological complete response (pCR) on treatment outcome.

Methods: All patients treated with nRCT followed by surgery between January 2005 and December 2015 were reviewed. The patients were categorized into two groups according to the pathological response following nRCT: pCR group and non-pCR group.

Results: Fifty-six patients with invasive cancer, 23 patients (41.1\%) achieved pCR and 33 patients had nonpCR $(58.9 \%)$ following nRCT. The average age was 62 years $( \pm 9.1)$, and most patients were males $(83.9 \%)$. Histological types included squamous cell carcinoma (75\%) and adenocarcinoma (25\%). The total radiation dose was 45 Gy in $76.8 \%$ of the patients and 50.4 Gy in $23.2 \%$. The median overall survival (OS) of the entire group was $3.5 \pm 1.2$ years, and the 5-year OS rate was 38.2\%, while the median disease-free survival (DFS) was $2.1 \pm 0.4$ years and the 5 -year DFS rate was $33.1 \%$. The patients who achieved pCR had significantly higher 5-year OS and 5-year DFS rates: $47.2 \%$ and $48 \%$ compared to $27.3 \%$ and $21 \%$ for the non-pCR patients respectively $(\mathrm{P}=0.04,0.03)$. The median time of local recurrence was $3.8 \pm 0.4$ years in pCR group and $1.8 \pm 0.2$ years in non-pCR group $(\mathrm{P}=0.01)$, while the median time of distant metastases in pCR group was $1.2 \pm 0.5$ years and $1.1 \pm 0.2$ years in non-pCR group $(\mathrm{P}=0.6)$.

Conclusions: Complete pathological response predicts significantly higher rates of OS and DFS in patients with locally advanced esophageal cancer treated with nRCT followed by surgery.

Keywords: Pathological complete response (pCR); neoadjuvant treatment; neoadjuvant radiochemotherapy (nRCT); esophageal cancer

Submitted Feb 14, 2018. Accepted for publication May 27, 2018.

doi: $10.21037 /$ jtd.2018.06.85

View this article at: http://dx.doi.org/10.21037/jtd.2018.06.85

\section{Introduction}

Neoadjuvant radiochemotherapy (nRCT) has been the standard of care in the context of a curative treatment strategy of non-metastatic but locally advanced esophageal cancer. The addition of nRCT to the surgery might be associated with improvement of overall survival (OS) and disease-free survival (DFS) in this group. The trimodal strategy, encompassing surgery, radiotherapy and chemotherapy, and its clinical meaning has been widely 
studied and has become a standard of care (1).

Several meta-analyses have found an OS benefit of the application of nRCT prior to surgery compared surgery alone (2-4). The Dutch controlled randomized trial CROSS has showed a clear survival benefit of nRCT and, consecutively, the regimen, encompassing of deescalated radiotherapy dose (41.4 Gy) and a well tolerable chemotherapy combination (carboplatin, paclitaxel) has become a new treatment option for the patients with locally advanced esophageal cancer in many institutes around the world $(5,6)$.

There are, still, biological factors influencing survival outcome that are in urgent need to be studied and understood. The role of the pathologic response to nRCT might be one clinically significant component in this puzzle. It could give useful information for the prediction of treatment outcome and patterns of disease recurrence. Pathologic response also provides valuable in-vivo treatment related information enlightening the further treatment decisions $(5,7)$. Recently, pathological complete response (pCR) following nRCT for esophageal cancer is gaining much attention and it was the primary end point in one of the most recently published randomized studies (NEOSCOPE) comparing two different regimens of nRCT (8).

Because of this necessity, we initiated a clinical study in the context of comparative effectiveness research. In this study, we assessed the impact of pCR on the treatment outcome, specifically OS and DFS, in patients with locally advanced esophageal cancer treated with nRCT and surgery.

\section{Methods}

\section{Patients}

All medical records of the patients diagnosed with esophageal cancer at any stage from January 2005 to December 2015 were reviewed. We found a total of 243 patients; only patients with respectable locally advanced esophageal cancer who were curatively treated with nRCT followed by surgery were included in the analyses. A multidisciplinary team consisting of experienced consultant surgeons, radiation and medical oncologists have discussed each of the analyzed individual cases. Ultimately, a total of 56 patients who matched the inclusion criteria were selected for the analysis and stratified according to the pathological response in the operative specimen into two groups: patients with pCR (pCR group) versus patients with non-pCR (nonpCR group).

\section{Preoperative workup}

The initial evaluation for staging included variable imaging modalities as well as endoscopic examination. Contrast enhanced computed tomography (CT) and endoscopic ultrasonography (EUS) were performed for all the patients. Positron emission tomography (PET)-CT was performed when necessary. All the patients were staged pretreatment according to the TNM staging system of the Union for International Cancer Control (UICC), sixth edition.

\section{$n R C T$}

The radiation therapy was planned using thin-slice CT images. Gross tumor volume (GTV) was defined according to the diagnostic CT scan, endoscopy, EUS, and PETCT (when available), clinical target volume (CTV) was generated by extending the GTV 2-3 cm superiorlyinferiorly and $1-1.5 \mathrm{~cm}$ radially, excluding the nearby organs except when infiltrated. Planning target volume (PTV) was automatically generated by extending the CTV $1 \mathrm{~cm}$. The daily radiation dose was 1.8 Gy in 25 or 28 fractions delivered by $3 \mathrm{D}$-conformal (in most cases) or by intensity modulated radiation treatment (IMRT). Simultaneous weekly chemotherapy regimens were documented and grouped.

\section{Surgery}

Surgery was performed 6-8 weeks after finishing the nRCT. Esophagectomy and standard regional lymphnode dissection were performed through transhiatal (open or minimally invasive), transthoracic, or three-incision approach, according to the location of the tumor.

\section{Pathologic examination}

Three different grading systems were used to document the pathological response following nRCT: Mandard et al. (9), Becker et al. (10), and Schneider et al. (11).

Complete regression was defined in the three systems as the absence of any viable tumor cells in the primary tumor location as well as in the regional lymph nodes (Table 1).

\section{Statistical analysis}

Results were reported as mean \pm standard error of mean, median with range, or as absolute value with the 
Table 1 Classification of the different grading systems used to document the pathological response

\begin{tabular}{ll}
\hline Description & Corresponding grade \\
\hline $\begin{array}{l}\text { Minimal regression or no } \\
\text { regression }\end{array}$ & $\begin{array}{l}\text { Schneider I; Becker 3; } \\
\text { Mandard 4/5 }\end{array}$ \\
Middle grade regression & $\begin{array}{l}\text { Schneider II; Becker 2; } \\
\text { Mandard 3 }\end{array}$ \\
High-grade regression & Schneider III; Becker 1b; \\
& Mandard 2 \\
Complete regression (no viable & Schneider IV; Becker 1a; \\
tumor cells) & Mandard 1 \\
\hline
\end{tabular}

corresponding percentage as indicated. The $t$-test was performed to compare sample mean of continuous variables. Categorical variables were compared by the Chi-square test. Survival analysis was performed using Kaplan-Meier analysis and log-rank test. Statistical significance level was established at $\mathrm{P}<0.05$. Follow-up duration was calculated starting from the date of surgery to the date of the last available follow-up visit. Statistical analyses were performed using IBM SPSS Statistics for Windows, Version 22.0 (IBM Corp., Armonk, NY, USA).

\section{Ethical considerations}

The institutional Ethic Committee of Humboldt University Berlin (Charité) (No. 1506) approved the study before starting the starting the data collection and analyses.

\section{Results}

\section{Patient and tumor characteristics}

A total of 56 patients who matched the inclusion criteria were included in the analysis, of them $23(41.1 \%)$ had pCR and 33 (58.9\%) had non-pCR. Patient and tumor characteristics are summarized in Table 2.

Most patients were males $(83.9 \%)$ with mean age of 63 years, there was similar sex and age distribution between both pCR and non-pCR groups.

Among the pCR group, middle esophagus was the most frequent tumor location (52.2\%), while in non-pCR group distal tumors were most frequently encountered (60.6\%). Most patients in each group had squamous cell carcinoma (73.9\% pCR; 75.8\% non-pCR). In both pCR and non-pCR groups, tumors were moderately differentiated in $56.5 \%$ and $57.6 \%$ of the patients respectively.
As regards the initial clinical staging in both groups, $>80 \%$ of patients had T3 or T4 tumors, and $>75 \%$ had N+ disease. Most patients had advanced stage disease (stage III/ IV): $69.5 \%$ in pCR group and $66.7 \%$ in non-pCR group.

\section{$n R C T$}

Most of patients in both groups had total radiation dose of 45 Gy (82.6\% pCR; $72.7 \%$ non-pCR). Details of the nRCT are summarized in Table 3.

\section{Surgery}

All patients underwent esophagectomy within 8 weeks after the finishing nRCT. Most patients were operated via Transhiatal approach (73.9\% pCR; 66.6\% non-pCR). No post-operative mortality occurred in both groups. Minimal post-operative morbidity recorded, Table 4 .

\section{Follow-up duration}

The median follow-up duration for the whole study cohort was 1.6 years (range, $0.1-8.7$ years), in the pCR group, it was 1.8 years (range, $0.1-8.7$ years), while in the non-pCR group it was 1.5 years (range, $0.6-7.3$ years).

\section{Local recurrence and distant metastases}

There were 6 local recurrences in the pCR group (26.1\%) and 13 local recurrences in the non-pCR group (39.4\%). The median time to local recurrence was significantly longer in pCR group; $3.8 \pm 0.4$ versus $1.8 \pm 0.2$ years in nonpCR group, $\mathrm{P}=0.01$.

Six distant metastases events were recorded in the pCR group $(26.1 \%)$ versus 17 events in the non-pCR group (51.5\%). The median time to distant metastases was similar in the two groups: $1.2 \pm 0.5$ and $1.1 \pm 0.2$ years for the pCR group and non-pCR group respectively, $\mathrm{P}=0.6$.

\section{Survival}

OS of the entire patients was $3.5 \pm 1.2$ years, and the 5 -year OS rate was $38.2 \%$. Patients who achieved pCR had significantly higher median OS and 5-year OS: $4.1 \pm 1.1$ years and $47.2 \%$ compared to $1.7 \pm 0.7$ years and $27.3 \%$ for patients who did not achieve $\mathrm{pCR}(\mathrm{P}=0.04)$ (Figure 1).

As regards the median DFS, it was $2.1 \pm 0.4$ years and the 5 -year DFS rate was $33.1 \%$ for all patients. In the pCR 
Table 2 Patients and disease characteristics

\begin{tabular}{|c|c|c|c|c|c|}
\hline Characteristics & Category & All patients $(\mathrm{N}=56), \mathrm{n}(\%)$ & $\mathrm{pCR}(\mathrm{N}=23), \mathrm{n}(\%)$ & Non-pCR (N=33), n (\%) & $\mathrm{P}$ \\
\hline Sex & Female & $9(16.1)$ & $4(17.4)$ & $5(15.2)$ & \\
\hline Age (year) & Mean (SEM) & $62 \pm 9.1$ & $63.5 \pm 1.8$ & $60.7 \pm 1.7$ & 0.26 \\
\hline \multirow[t]{2}{*}{ Location } & Proximal & $5(8.9)$ & $3(13.1)$ & $2(6.1)$ & 0.16 \\
\hline & Distal & $28(50)$ & $8(34.8)$ & $20(60.6)$ & \\
\hline \multirow[t]{2}{*}{ Histology } & Squamous cell carcinoma & $42(75)$ & $17(73.9)$ & $25(75.8)$ & 0.87 \\
\hline & Adenocarcinoma & $14(25)$ & $6(26.1)$ & $8(24.2)$ & \\
\hline Grade & Well & $5(8.9)$ & $2(8.7)$ & $3(9.1)$ & 0.37 \\
\hline \multirow[t]{4}{*}{ cT stage } & $\mathrm{cT} 1$ & $1(1.8)$ & $0(0)$ & $1(3.0)$ & 0.48 \\
\hline & сT2 & $8(14.3)$ & $2(8.7)$ & $6(18.2)$ & \\
\hline & cT3 & $44(78.6)$ & $19(82.6)$ & $25(75.8)$ & \\
\hline & cT4 & $3(5.4)$ & $2(8.7)$ & $1(3.0)$ & \\
\hline \multirow[t]{4}{*}{ cN stage } & NO & $14(25.0)$ & $4(17.4)$ & $10(30.3)$ & 0.54 \\
\hline & N1 & $31(55.4)$ & $14(60.9)$ & $17(51.5)$ & \\
\hline & N2 & $10(17.9)$ & $5(21.7)$ & $5(15.2)$ & \\
\hline & N3 & $1(1.8)$ & $0(0)$ & $1(3.0)$ & \\
\hline \multirow{5}{*}{ Clinical stage } & IIA, T2-3NOMO & $12(21.4)$ & $5(21.7)$ & $7(21.2)$ & \\
\hline & IIB, T1-2N1M0 & $5(8.9)$ & $2(8.7)$ & $3(9.1)$ & \\
\hline & III, T3N1M0, T4 any N M0 & $35(62.5)$ & $15(65.2)$ & $20(60.6)$ & \\
\hline & IVA, M1a & $3(5.4)$ & $1(4.3)$ & $2(6.1)$ & \\
\hline & IVB, M1b & $0(0.0)$ & $0(0)$ & $0(0)$ & \\
\hline
\end{tabular}

SEM, standard error of mean.

group, the median DFS was 3.1 \pm 0.6 years and the 5 -year DFS was $48 \%$ versus $1.1 \pm 0.3$ years and $21 \%$ in the nonpCR group respectively $(\mathrm{P}=0.03)$ (Figure 1).

\section{Discussion}

There are different biological factors that influence the treatment outcome in patients with resectable locally advanced esophageal cancer. Full analysis of these factors is important in order to understand the role of the trimodal treatment and its effects on OS and DFS. One important factor is the pCR which through extensive study of its impact on the treatment outcome could change the treatment options for a subset of the patients. 
Table 3 Details of neoadjuvant radiochemotherapy

\begin{tabular}{|c|c|c|c|c|c|}
\hline Treatment & Category & All patients $(\mathrm{N}=56)(\%)$ & $\mathrm{pCR}(\mathrm{N}=23)(\%)$ & Non-pCR (N=33) (\%) & $\mathrm{P}$ \\
\hline Radiation total dose & Total dose 50.4 Gy & $13(23.2)$ & $4(17.4)$ & $9(27.3)$ & \\
\hline \multirow[t]{2}{*}{ Radiation technique } & 3D-conformal planning & $37(66.1)$ & $15(65.2)$ & $22(66.7)$ & 0.53 \\
\hline & IMRT & $19(33.9)$ & $8(34.8)$ & $11(33.3)$ & \\
\hline \multirow{2}{*}{ Neoadjuvant CTx } & Carboplatin + paclitaxel & $21(37.5)$ & $10(43.5)$ & $11(33.3)$ & \\
\hline & Other & $7(12.5)$ & $3(13.0)$ & $4(12.1)$ & \\
\hline
\end{tabular}

IMRT, intensity modulated radiation therapy; 5-FU, 5-fluorouracil.

Table 4 Surgical results

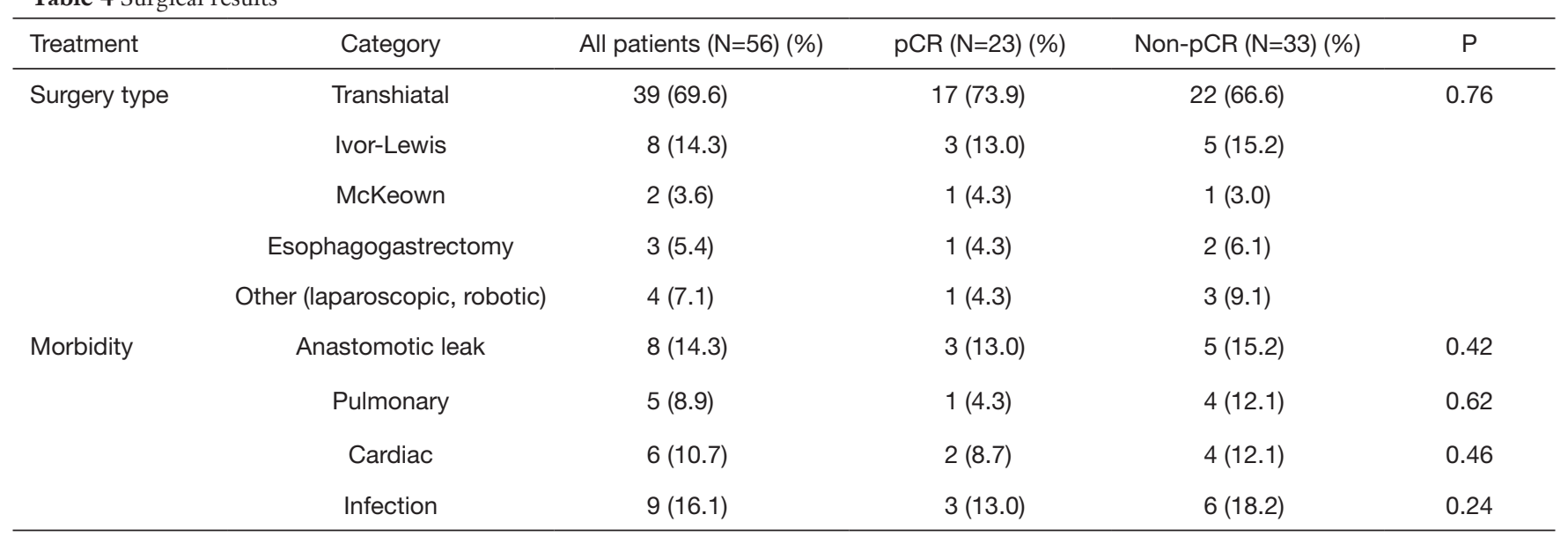
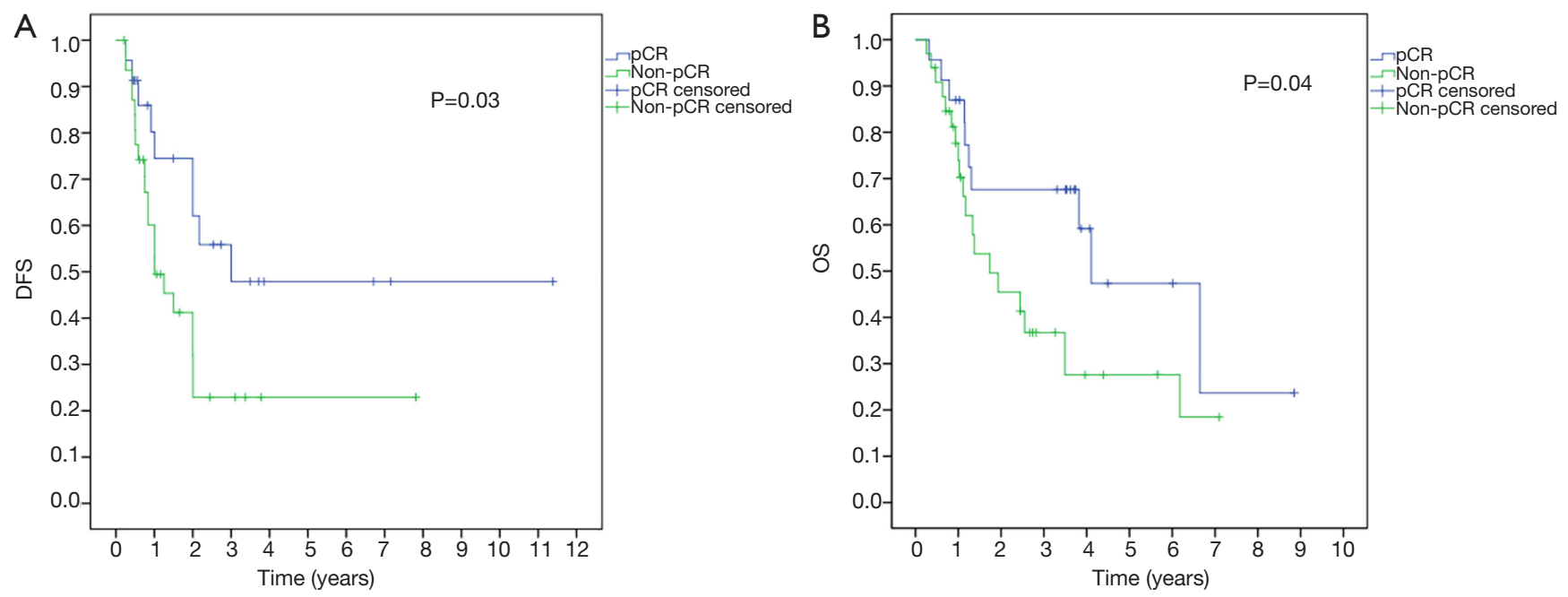

Figure 1 Kaplan-Meier estimates of DFS (A) and OS (B). DFS, disease-free survival; OS, overall survival. 
Pathologic response to neoadjuvant treatment in cancer esophagus has been repeatedly studied. Many studies have implied the potential benefits of neoadjuvant therapy other than improvement in survival, in-vivo assessment of the biological behavior of the tumor cells could help in further clinical decisions. If the tumor progressed after neoadjuvant therapy or clinically achieved complete response, the decision of surgery could be revised. Furthermore, in the patients who develop disease relapse after surgery, the initial response to neoadjuvant treatment could guide the further treatment (6,12-14).

In our study, we found 23 patients $(41.1 \%)$ with pCR out of total 56 patients who received nRCT. The rate of pCR is comparable to what has been documented by other studies which reported pCR ranging between $26 \%$ and $40.4 \%(5,15-18)$. In the Dutch CROSS trial, the rate of pCR in the nRCT arm was $29 \%$ while it was significantly higher among the patients with squamous cell carcinoma reaching $49 \%$ versus $23 \%$ in patients with adenocarcinoma ( $\mathrm{P}=0.008)$. Meredith et al. reported $40.4 \%$ overall rate of pCR following nRCT with no difference between squamous cell carcinoma and adenocarcinoma (18). We found a similar rate of pCR among patients with squamous cell carcinoma $(6 / 14 ; 42.9 \%)$ and patients with adeno carcinoma (17/42; 40.5\%).

Prediction of pathological response to neoadjuvant treatment using PET-CT remains controversial; the sensitivity of PET-CT in detecting the therapeutic response was not constant among studies and ranged between $61.8 \%$ and $93 \%$, local tissue inflammation following the radiation therapy may (18-20). In our study, PET-CT imaging before the scheduled surgery was regularly performed and the response to the nRCT was documented only based on the histopathological examination of the surgical specimen.

We did not find a significant difference in the median time to develop distant metastases between the pCR group and the non-pCR group, although the total events of distant metastases at the last follow-up visit were higher in patients who had residual disease following $\mathrm{nRCT}$ in comparison to the patients who had pCR, $51.5 \%$ versus $26.6 \%$ respectively. The median time to develop distant metastasis was similar between the pCR group and the nonpCR group, 1.2 and 1.1 years respectively $(\mathrm{P}=0.6)$.

Patients in the pCR group had fewer local recurrences than the patients in non-pCR group, $26.1 \%$ and $39.4 \%$ respectively. The median time to local recurrence among the patients in the pCR group was 3.8 years which is significantly longer than the corresponding time in the
non-pCR group (1.8 years). Meredith et al. found lower recurrence rate in patients who achieve $\mathrm{pCR}$ and concluded that, the patients who achieved pCR were less likely to develop disease recurrence than patients who had disease residual following neoadjuvant therapy (18).

Neoadjuvant RCT followed by surgery has yielded significant survival benefit when compared to surgery alone as reported by many studies $(5,15-18)$, The Dutch CROSS trial found $47 \% 5$-year OS in the nRCT arm, which is higher than our reported 5-year OS rate for the entire patients $(38.2 \%)$. Other studies reported similar rates (33-39.4\%) $(16,18)$.

Our reported 5 -year OS is slightly inferior to what has been reported by the CROSS study, this could be attributed to the heterogeneity in the radiation dose, the type of simultaneous chemotherapy, and the surgical technique used in our study. The exact impact of these heterogeneity on treatment outcome could not be statistically proved due to limitations of the patient's number.

Berger et al. analyzed the treatment outcome in patients achieving pCR following nRCT and reported $48 \%$ 5 -year OS rate and 50-month median OS time (16), while Donahue et al. and Meredith et al. reported 55\% and 52\% 5 -year OS rate in patients who had pCR in the surgical specimens following nRCT respectively $(15,18)$. The three studies founded a significant OS benefit among patients who achieved pCR compared with patients who did not, these results compare favorably with our reported OS data. We found 47.2\% 5-year OS in patients who achieved pCR versus $27.3 \% 5$-year $\mathrm{OS}$ in patients who did not $(\mathrm{P}=0.04)$, the median OS time was 4.1 years (49.2 months) versus 1.7 years (20.4 months) respectively.

In one study, the OS benefit in patients who achieve pCR was persistent when the patients were stratified according to initial disease stage, or according to histologic type (18), due to the limited number of the patients in our study, similar sub-analyses were not possible.

The 5 -year DFS was $48 \%$ for the pCR group and $21 \%$ for the non-pCR group $(\mathrm{P}=0.03)$, this significant difference was also consistent in two similar studies who reported 5 -year DFS for patients who achieved pCR of $49 \%$ and $52 \%$ versus $25.4 \%$ and $19 \%$ for the patients who did not respectively $(15,18)$.

As shown in our data analyses as well as by other studies, a significant survival benefit and better treatment outcome are connected to the complete pathological response to neoadjuvant RCT.

Based on our results as well as the results from other 
similar studies; there were no solid identifiable patientrelated or treatment-related factors that could predict the probability of obtaining pCR following nRCT. Therefore, identifying certain biomarkers that could predict the efficacy of nRCT remains essential, as it could direct the selection of the treatment options for different groups of patients. One study has linked the overexpression of epidermal growth factor receptor (EGFR) in $59.6-76 \%$ of squamous cell carcinoma patients, which is associated with a poor prognosis (21). Other study suggested a link between the downregulation of the microRNA-330-5p protein and the poor response to the nRCT in adenocarcinoma patients (22). A recently introduced prediction model based on combining the clinical T-stage with the radiomic features of the baseline 18F-FDG PET imaging showed promising results regarding prediction of the response to the nRCT (23).

For further discussions, one might mention three implications. First, trimodal strategies seem to be effective and safe in the treatment of patients with resectable locally advanced esophageal cancer. Leading to the second question, which would include the notion of de-escalation in the extent of radiotherapy and chemotherapy. Third, with more caution but inevitably, another question would rise: will be a subgroup of patients identifiable, in which the surgery might be omitted? Not surprisingly, two ongoing trials investigate the value of primary esophagectomy in patients with clinical complete response to nRCT according to the CROSS study $(24,25)$. The accuracy in detecting the response to $\mathrm{nRCT}$ remains the major point of criticism in such protocols. Nevertheless, the results of these studies could have an impact on individualizing the treatment options.

\section{Conclusions}

This clinical study, performed in the context of comparative effectiveness research, demonstrates that $\mathrm{pCR}$ after nRCT in patients with locally advanced esophageal cancer predicts significantly higher rates of survival and generally good treatment outcome. This fact could aid in defining future treatment options in the case of disease recurrence, and could justify further prospective studies investigating the chances to change or omit surgical procedures in order to avoid or delay the risks.

\section{Acknowledgements}

None.

\section{Footnote}

Conflicts of Interest: The authors have no conflicts of interest to declare.

Ethical Statement: The institutional Ethic Committee of Humboldt University Berlin (Charité) (No. 1506) approved the study before starting the data collection and analyses. Written informed consent was obtained from the patients for publication of this manuscript and any accompanying images.

\section{References}

1. Herskovic A, Martz K, Al-Sarraf M, et al. Combined Chemotherapy and Radiotherapy Compared with Radiotherapy Alone in Patients with Cancer of the Esophagus. N Engl J Med 1992;326:1593-8.

2. Gebski V, Burmeister B, Smithers BM, et al. Survival benefits from neoadjuvant chemoradiotherapy or chemotherapy in oesophageal carcinoma: a meta-analysis. Lancet Oncol 2007;8:226-34.

3. Sjoquist KM, Burmeister BH, Smithers BM, et al. Survival after neoadjuvant chemotherapy or chemoradiotherapy for resectable oesophageal carcinoma: an updated metaanalysis. Lancet Oncol 2011;12:681-92.

4. Zhang CD, Zeng YJ, Li HW, et al. Neoadjuvant chemotherapy for nonmetastatic esophago-gastric adenocarcinomas: a systematic review and meta-analysis. Cancer Invest 2013;31:421-31.

5. van Hagen P, Hulshof MC, van Lanschot JJ, et al. Preoperative Chemoradiotherapy for Esophageal or Junctional Cancer. N Engl J Med 2012;366:2074-84.

6. Shapiro J, van Lanschot JJ, Hulshof MC, et al. Neoadjuvant chemoradiotherapy plus surgery versus surgery alone for oesophageal or junctional cancer (CROSS): long-term results of a randomised controlled trial. Lancet Oncol 2015;16:1090-8.

7. Meguid RA, Hooker CM, Taylor JT, et al. Recurrence after neoadjuvant chemoradiation and surgery for esophageal cancer: Does the pattern of recurrence differ for patients with complete response and those with partial or no response? J Thorac Cardiovasc Surg 2009;138:1309-17.

8. Mukherjee S, Hurt CN, Gwynne S, et al. NEOSCOPE: A randomised phase II study of induction chemotherapy followed by oxaliplatin/capecitabine or carboplatin/ paclitaxel based pre-operative chemoradiation for 
resectable oesophageal adenocarcinoma. Eur J Cancer 2017;74:38-46.

9. Mandard AM, Dalibard F, Mandard JC, et al. Pathologic assessment of tumor regression after preoperative chemoradiotherapy of esophageal carcinoma. Clinicopathologic correlations. Cancer 1994;73:2680-6.

10. Becker K, Mueller JD, Schulmacher C, et al. Histomorphology and grading of regression in gastric carcinoma treated with neoadjuvant chemotherapy. Cancer 2003;98:1521-30.

11. Schneider PM, Baldus SE, Metzger R, et al. Histomorphologic tumor regression and lymph node metastases determine prognosis following neoadjuvant radiochemotherapy for esophageal cancer: implications for response classification. Ann Surg 2005;242:684-92.

12. Cunningham D, Allum WH, Stenning SP, et al. Perioperative chemotherapy versus surgery alone for resectable gastroesophageal cancer. $\mathrm{N}$ Engl J Med 2006;355:11-20.

13. Ychou M, Boige V, Pignon JP, et al. Perioperative chemotherapy compared with surgery alone for resectable gastroesophageal adenocarcinoma: an FNCLCC and FFCD multicenter phase III trial. J Clin Oncol 2011;29:1715-21.

14. Allum WH, Stenning SP, Bancewicz J, et al. Long-term results of a randomized trial of surgery with or without preoperative chemotherapy in esophageal cancer. J Clin Oncol 2009;27:5062-7.

15. Donahue JM, Nichols FC, Li Z, et al. Complete pathologic response after neoadjuvant chemoradiotherapy for esophageal cancer is associated with enhanced survival. Ann Thorac Surg 2009;87:392-8; discussion 398-9.

16. Berger AC, Farma J, Scott WJ, et al. Complete response to neoadjuvant chemoradiotherapy in esophageal carcinoma is associated with significantly improved survival. J Clin Oncol 2005;23:4330-7.

17. Tiesi G, Park W, Gunder M, et al. Long-term survival

Cite this article as: Soror T, Kho G, Zhao KL, Ismail M, Badakhshi H. Impact of pathological complete response following neoadjuvant chemoradiotherapy in esophageal cancer. J Thorac Dis 2018;10(7):4069-4076. doi:10.21037/jtd.2018.06.85 based on pathologic response to neoadjuvant therapy in esophageal cancer. J Surg Res 2017;216:65-72.

18. Meredith KL, Weber JM, Turaga KK, et al. Pathologic response after neoadjuvant therapy is the major determinant of survival in patients with esophageal cancer. Ann Surg Oncol 2010;17:1159-67.

19. Luketich JD, Schauer PR, Meltzer CC, et al. Role of Positron Emission Tomography in Staging Esophageal Cancer. Ann Thorac Surg 1997;64:765-9.

20. Weber WA, Ott K, Becker K, et al. Prediction of Response to Preoperative Chemotherapy in Adenocarcinomas of the Esophagogastric Junction by Metabolic Imaging. J Clin Oncol 2001;19:3058-65.

21. Zhang W, Zhu H, Liu X, et al. Epidermal growth factor receptor is a prognosis predictor in patients with esophageal squamous cell carcinoma. Ann Thorac Surg 2014;98:513-9.

22. Bibby BA, Reynolds JV, Maher SG. MicroRNA-330-5p as a Putative Modulator of Neoadjuvant Chemoradiotherapy Sensitivity in Oesophageal Adenocarcinoma. PLoS One 2015;10:e0134180.

23. Beukinga RJ, Hulshoff JB, Mul VEM, et al. Prediction of Response to Neoadjuvant Chemotherapy and Radiation Therapy with Baseline and Restaging 18F-FDG PET Imaging Biomarkers in Patients with Esophageal Cancer. Radiology 2018;287:983-92.

24. Noordman BJ, Shapiro J, Spaander MC, et al. Accuracy of Detecting Residual Disease After Cross Neoadjuvant Chemoradiotherapy for Esophageal Cancer (preSANO Trial): Rationale and Protocol. JMIR Res Protoc 2015;4:e79.

25. Centre Hospitalier Universitaire Dijon. Comparison of Systematic Surgery Versus Surveillance and Rescue Surgery in Operable Oesophageal Cancer With a Complete Clinical Response to Radiochemotherapy (Esostrate). ClinicalTrials.gov Identifier NCT02551458, 2015. 